\title{
Flow Simulation And Performance Analysis Of Centrifugal Compressor Using Cfd_Tool
}

\author{
${ }^{1}$ Tesfaye Barza ${ }^{2}$ G.Lakshmikanth \\ ${ }^{1}$ Asst. Lecturer, Dept.of Mechanical Engg(Thermal), Wolaita Sodo University, Ethiopia \\ E-Mail:tesfayebarza@gmail.com \\ ${ }^{2}$ Asst. Professor, Dept. of Mechanical Engg (Manufacturing), Wolaita Sodo University, Ethiopia \\ E-Mail:lakshmikanthlaksh@gmail.com
}

\begin{abstract}
This paper is concerned the flow simulation and performance analysis of the Centrifugal Compressor Using CFD Tool. The complex internal flow of centrifugal compressor can be well analyzed, and the unique design system needs to be developed. It should be early to use the interface and also flexible for input and output. A 3-D flow simulation of turbulent - fluid flow is presented to visualize the flow pattern in-terms of velocity, streamline and pressure distribution on the blade surface are graphically interpreted. The standard K- e turbulence model and the simple model algorithm were chosen for turbulence model and pressure distribution well determined. The simulation was steady Heat transfer and moving reference frame was used to consider the impeller interaction under high resolution. Furthermore, A computational Fluid Dynamics (CFD) 3-D simulation is done to analyze the impeller head and efficiency required of centrifugal compressor. The impeller is rotated for a constant revolution and mass flow rate, in this study initially the geometry of centrifugal compressor impeller is created by an ANSYS Vista CCD, and the Blade modeller done by Bladegen, Finally, CFD analysis was performed in ANSYS CFX using the ANSYS Turbo grid meshing tool. According to the analysis, as the number of impeller blades increases, so does the value of the head and power imparted, as well as the impeller's efficiency.
\end{abstract}

Keywords: ANSYS CFx, Centrifugal Compressor, Impeller, ANSYS BladeGEN, Performance Analysis, CFD

\section{INTRODUTION}

The centrifugal compressors have an extensive variety of applications particularly for power plants for small aircraft and helicopters, in process industries, compression of gases and vapours, because they can provide high-pressure ratios and large operating ranges with relatively high (isentropic) efficiencies as well as high discharge operations because they work at a constant rate. Centrifugal compressors mainly increase the kinetic energy of the fluid with in using a rotating impeller [1]. It is a mechanical device which is able to transfer the energy efficiently to the fluid medium so that it can be delivered in large quantities at several multiples of inlet pressure [2]. Computational Fluid Dynamics (CFD), which is one of the branches of fluid mechanics that uses numerical method sand algorithms to solve and analyze problems of fluid flows. Computers are used to perform the calculations required to simulate the interaction of liquids and gases with surfaces defined by boundary condition [3].Computational Fluid Dynamics (CFD) has grown from a mathematical curiosity to become an essential tool in almost every branch of fluid dynamics, from aerospace propulsion to weather prediction. CFD is commonly accepted as referring to the board topic encompassing the numerical solution, by computational methods, of the governing equations which describe fluid flow, the set of the Navier-Stokes equations, continuity and any additional conservation equations, for example energy or species concentrations [4].

In addition, CFD fluid analysis software predicts the interaction of working fluids with their geometry and operating environment. To accurately predict these interactions, you need to understand the energy loss model embedded in the design code. These loss models show how much performance is degraded due to inherent or possibly inaccurate geometric and operational constraints. Such energy losses include skin friction, excessive pressure recovery, flow generation, flow re-circulation and blade tip leakage. Centrifugal compressors draw air through a central rotating wheel and accelerate the air at high speeds. High speeds flow radially outward through the bowl-shaped housing. After leaving the wheel, the airspeed slows down and velocity energy is converted to pressure. This type of 
compressor is a high speed device. It basically consist of a fixed housing that contains a rotating impeller that gives the air high speed. The air is drawn into the eyes of the impeller and swirls at high speed by the blades of the afferent disc. At each point of the airflow through the impeller, the centripetal acceleration is achieved by the print head, increasing the static pressure of the air from the eye towards the tip of the impeller. The memory of the increase in static pressure is in the diffuser, and the high speed of the air leaving the tip of the impeller decreases somewhere within the speed range where the air is causing the impeller eye. Note that some back pressure is lost due to the friction of the diffuser. As mentioned earlier, the stage simulation consists of two sections (impeller and diffuser) connected by a "stage type" mix level where the flow characteristics are averaged across the circumference. The exit of the impeller and the entrance of the diffuser form this interface. At the inlet, the boundary was defined as a subsonic inlet with the measured total temperature, total pressure, and flow direction profile. The degree of turbulence was defined as an average intensity of about $5 \%$. The periodic constraint was applied to the periodic impeller plane in the center of the impeller flow path. Blades, hubs and shrouds were defined as insulating walls with appropriate rotational speeds. .

Mainly this project is all about flow simulation and performance analysis of centrifugal compressor using CFD -tool. The objectives of this Flow simulation and Performance analysis of Centrifugal Compressor are as follows:

* To determine the flow simulation and performance analysis of centrifugal compressor

* To analyze the basic parameters ( pressure, temperature and velocity) and

* To determine the sound analysis(pressure, power, directivity and strength)

Aerodynamic and Preliminary Design Specifications by ANSYS Vista CCD mentioned as follows, The compressor is designed at sea level (101.325kPa and 288.15k) and the requirements are given in Table 1 and 2 below

Table 1. The design inputs and requirements at design point

\begin{tabular}{l|l|}
\hline Geometric Date of the original impeller & Specific value \\
\hline Pressure Ratio & $4.5 \mathrm{Kpa}$ \\
Mass flow rate & $3 \mathrm{~kg} / \mathrm{s}$ \\
Rotation speed & $40000 \mathrm{rpm}$ \\
Isotropic efficiency & 0.85 \\
\hline
\end{tabular}

Table 2. Main dimensions of the original impeller

\begin{tabular}{l|l|}
\hline Geometric Date of the original impeller & Dimensions \\
\hline Impeller outer diameter & $280 \mathrm{~mm}$ \\
Inducer hub diameter & $75 \mathrm{~mm}$ \\
Inducer tip diameter & $180 \mathrm{~mm}$ \\
Overall length & $140 \mathrm{~mm}$ \\
\hline
\end{tabular}

\begin{tabular}{l|l|}
\hline Other Geometric Date & Dimensions \\
\hline Vane hub inlet angle & $25^{0}$ \\
Shroud vane inlet angle & $60^{0}$ \\
Vane normal thickness & $1.5 \mathrm{~mm}$ \\
Shroud normal thickness & $0.5 \mathrm{~mm}$ \\
& \\
\hline
\end{tabular}




\section{METHODOLOGY}

\subsection{Geometry and Grid Generation}

The next stage is to generate the geometry after determining the velocity triangles and blade angles.ANSYS Workbench 16.0 was used throughout the design process in this investigation. Under the BladeGen the blade geometry (height, inducer hub diameter, exducer tip diameter, inlet and outlet blade angles, etc.) was formed in the ANSYS Vista CCD, whereas the required tip profile and tip clearance value was dictated to the tool.Blade thickness was also evaluated to assure that the required aerodynamic and structural standards were met.The blade's 2D profile is shown in the diagram below.

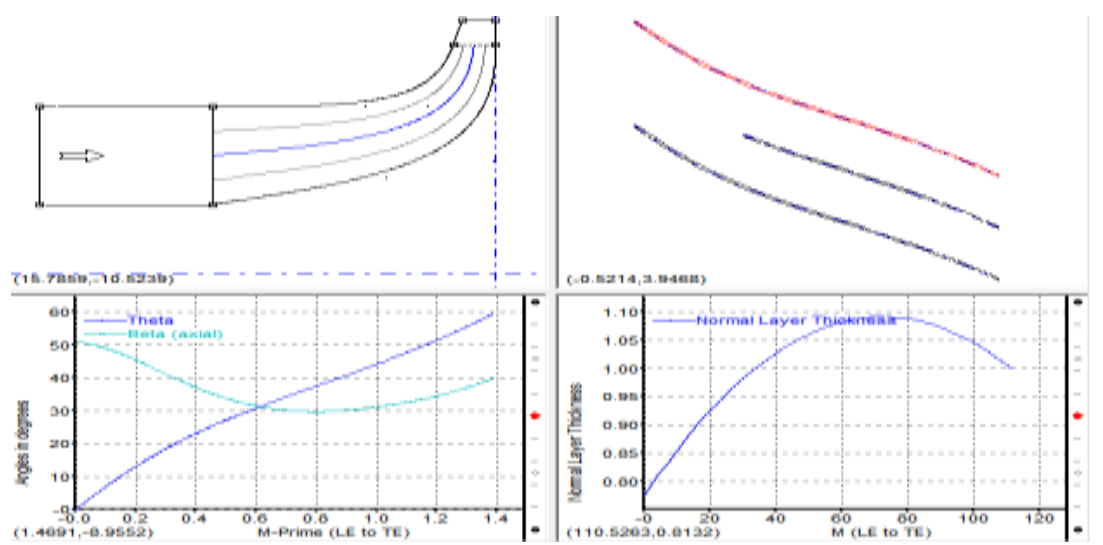

Figure 1. The ANSYS BladeGen view of the 2D blade profile

Here, the top left view which represents the Meridional profile of the blade generated under ANSYS bladege. The left base view is the blade angle distribution window. The base right window represents the blade thickness distribution; whereas the top right view is the blade-to blade view of the compressor. After the completion of the design process in BladeGen tool, the blade was divided into finite elements by using ANSYS Turbo-Grid. Here, by providing the enough number and quality of the solution grids, the accuracy of the solution is ensured. To determine the sufficient number After the completion of the design process in BladeGen tool, the blade was divided into finite elements by using ANSYS Turbo-Grid. Here, by providing the enough number and quality of the solution grids, the accuracy of the solution is ensured. To determine the sufficient number of grid cells, a mesh independence study was conducted and it was found out that approximately 900000 cells are enough to obtain an accurate solution.

\section{DOMAIN OF COMPUTATION AND GENERATION OF THE MESH}

Because of the variety of mesh generation methods, there are numerous ways to characterize a computing grid. In this section, we look at meshes created with the ANSYS Workbench software "Turbo-Grid."The set of topologies H / J / C / L-Grid is a first choice for the analysis of the centrifugal compressor impeller generated from the ANSYS Workbench program "CFX-BladeGEN."Figure below depicts the various boundaries for modeling a channel of the centrifugal compressor and the original turbo compressor impeller

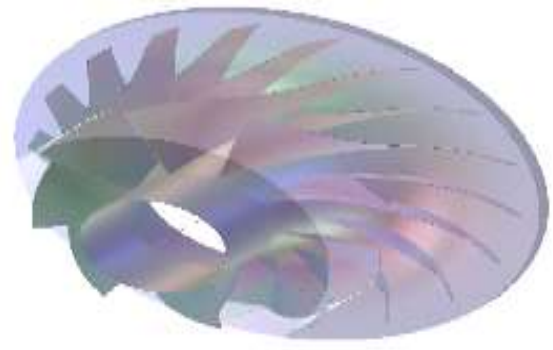

Figure 2 : shows a 3D view of the original turbo compressor impeller obtained from the ANSYS Workbench software "CFX-BladeGEN". 
The boundary conditions are required to set up for flow simulation in the turbulence model SST. The Fluid is turbulence in most of turbo machinery due to high Reynolds number. However, Reynolds number increases due to high density. ANSYS provides inbuilt module of Turbogrid which generates high quality hexahedral meshes for three dimensional fluid flow analyses. Three dimensional design from Blade GEN is then imported toTurbogrid for meshing. Shroud tip gap is provided in mm. A global size factor set 1 called as "Finemesh" around 420000 cells without boundary layer. Then calculated Reynolds number from 1D designis employed by the software to calculate the distribution of boundary layer mesh. Boundary layerrefinement can be done using cut-off edge to boundary layer factor. Near wall element size is fixed as absolute from calculated Reynolds Number. Completing all necessary tasks, finally3D meshes are generated with total number of element (Hexahedra)70230, total number of nodes 79980 and maximum edge length ratio $2.38999 \mathrm{e}-31$.

Table 3: Parameter considered in Meshing

\begin{tabular}{|l|l|}
\hline \multicolumn{2}{|c|}{ GENERAL PARAMETERS } \\
\hline Reynolds Number & $5^{*} 10^{7}$ \\
\hline Shroud Tip Mesh Method & Match Expansion at Blade Tip \\
\hline Topology Mesh Technique & ATM optimized \\
\hline Boundary Layer Refinement Control Factor & First Element offset \\
\hline Spanwise Blade distribution parameter & Proportional \\
\hline Hub Tip Mesh Method & Match Expansion at Blade Tip \\
\hline Near Wall Treatment & Absolute \\
\hline
\end{tabular}

\begin{tabular}{|l|l|}
\hline \multicolumn{2}{|c|}{ D2:CFx-CFx-Pre } \\
\hline Turbulence Numerics & High resolution \\
\hline Convergence Control & Max Iter-100 \\
\cline { 2 - 2 } & Min Iter-1 \\
\hline Convergence Criteria & RMS \\
\hline Equation Class & Continuity,Momentum\&Eddy Dissipation\&Turbulence KE \\
\hline Residual Target & $1 \mathrm{e}-4$ \\
\hline
\end{tabular}

(69.) Mesh Statistics

\begin{tabular}{|c|c|c|c|}
\hline Domain & \multicolumn{2}{|l|}{ ALL } & $\checkmark$ \\
\hline Mesh M & & Value & $\%$ Bad \\
\hline Minimun & Angle & $2.39858 \mathrm{e}-31$ & 0.0000 \\
\hline Maximu & Angle & $2.39875 \mathrm{e}-31$ & 0.0000 \\
\hline Maximu & ent Volume Ratio & $2.39915 e-31$ & 0.0000 \\
\hline Minimun & & $2.39914 e-31$ & 0.0000 \\
\hline Maximu & Length Ratio & $2.39899 \mathrm{e}-31$ & 0.0000 \\
\hline Maximu & nectivity Number & $2.39903 e-31$ & 0.0000 \\
\hline
\end{tabular}

Figure 3 : Mesh statics 
ANSYS -CFx provides the two models for turbulence namely: $k-\omega$ Shear stress model and $k-\varepsilon$ model. Wilcox initially proposed the k- $\omega$ model in 1988 (Wilcox, 1988). Menter had modified further changedto k- $\omega$ Shear stress model (Menter, 1994). k- $\varepsilon$ model was proposed by Launder and Spalding in 1972means older than k- $\omega$. This model is suitable for fully turbulent non-separated flows. It does not calculate very accurate flow field that exhibit adverse pressure gradient and strong curvature. Problem related to external body can be simulated more accurately with k- $\varepsilon$ model. k- $\omega$ shear stress model(SST) is recommended for accurate simulation in near wall treatment and internal flow like turbomachinery (Pecnik et al., 2012). SST model is chosen for the steady state flow simulation in centrifugal compressor. The Total pressure and temperature are defined at inlet with mass flow rate at outlet as boundarycondition shown in figure below

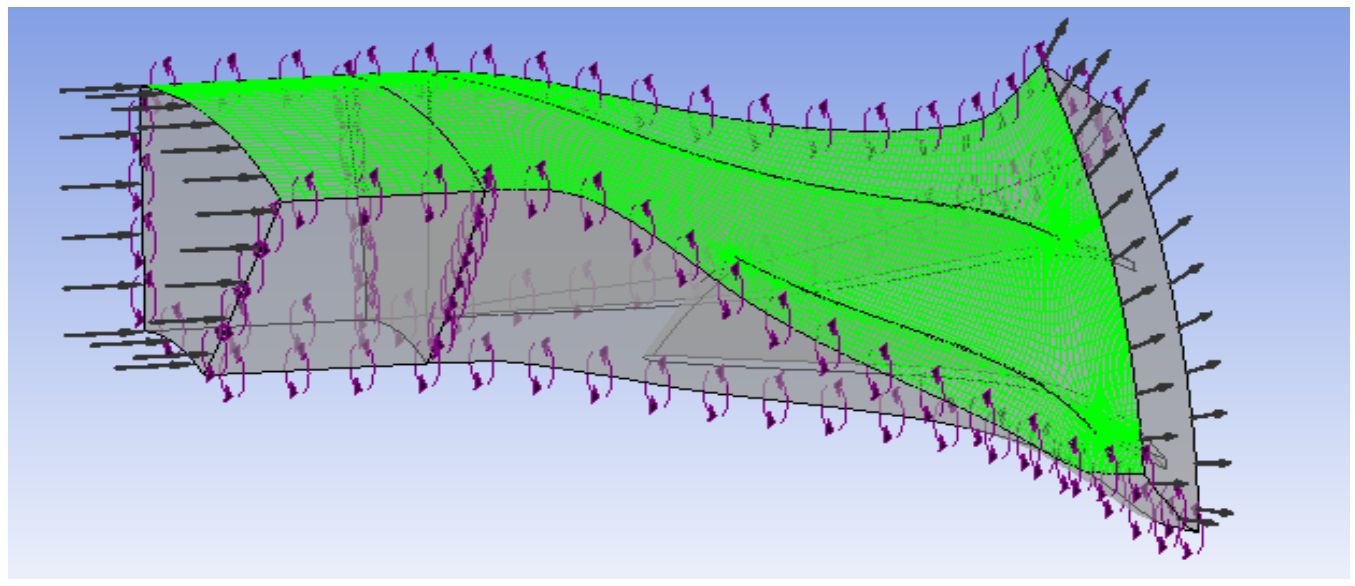

Figure 4: Boundary condition (D2: CFX-CFX-Pre)

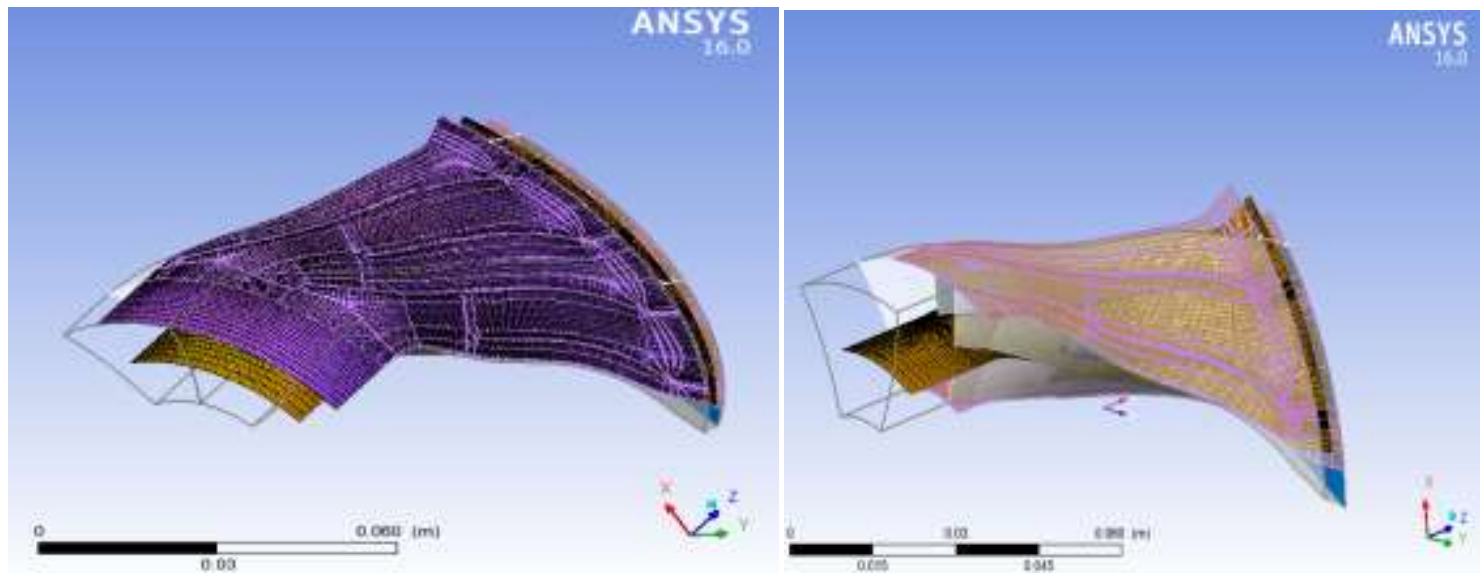

Figure 5 : 3-D Mesh Course With Reseanable Quality ( Topology Fully Suspended)

\section{ANALYSIS AND SIMULATION}

The simulation has proven to be well suited and efficiently accomplished. And the task analyzing the threedimensional structure of internal flows. In this work, two objectives will be follow educing CFDsoftware. Commercial software was used to create the CFD codes: CFX-BladeGEN and CFX-TurboGrid.CFX-Pre, CFXSolver, and CFX-Post can communicate via automatic or operator-controlled linkages.An compressible, steady-state analysis with an Epsilon, shear stress model was used in the simulation (SST) turbulence model and the Heat transfer (Total Energy) This type of analysis is robust and well-suited to applications with rotating components. 


\subsection{Static Pressure Contours at Span 0.5}

Figure 10 shows pressure contours in a blade-to-blade view at a span of 0.5.The contours indicate a gradual rise in pressure from the compressor's inlet to outlet due to the rotating impeller's dynamic action.At all tip clearances, a gradual increase in static pressure from input to output can be seen.Low pressure change is observed with PS on the blade's tip.Furthermore, without PS on the blade's tip, the pressure at the outflow is decreased.At all tip clearances, high pressure on the pressure side of the blade and low pressure on the suction side are observed.There is a reduction in pressure on both the pressure and suction sides when tip clearance is raised.

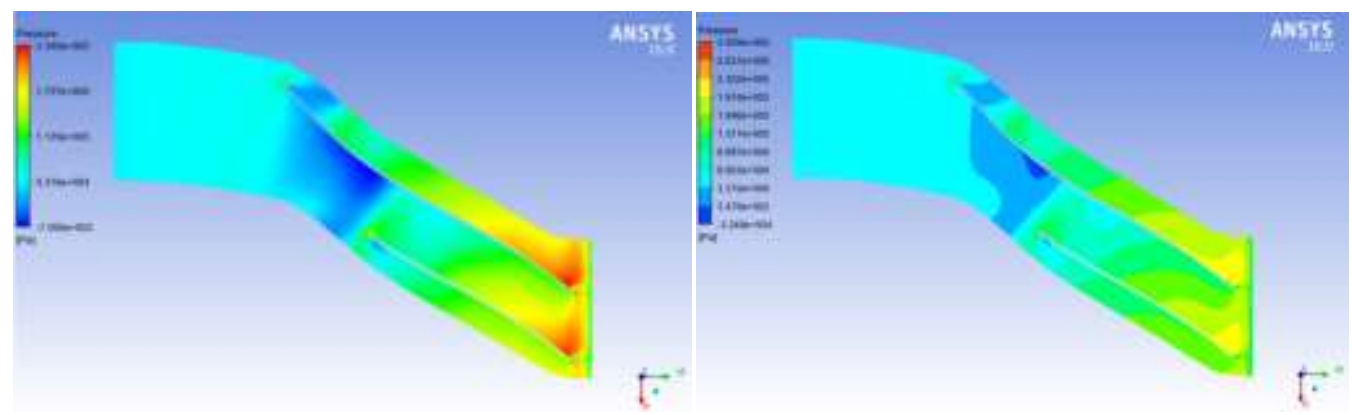

Figure 6: static Pressure contour without PS and with PS/ partial shroud

\subsection{Velocity Contours at Span 0.5}

Velocity contours in blade to blade view, at span 0.5 is shown in figure below. The contours show low velocity region on suction side of the blade. With PS on tip of the blade, the low velocity region is reducing and also velocity improvement is observed.

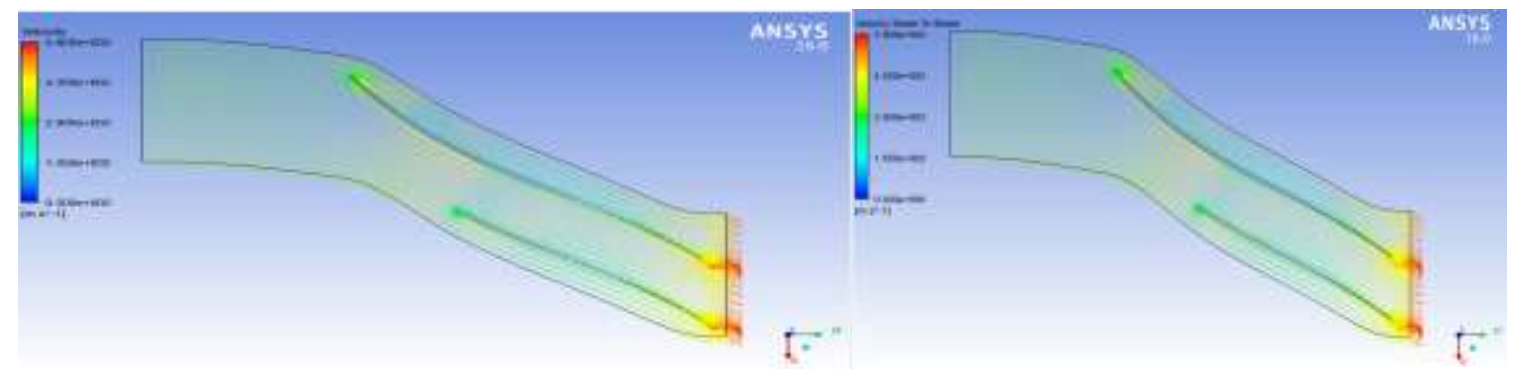

Figure.7 : Velocity contours for $2 . \%$ clearance for without PS and with PS

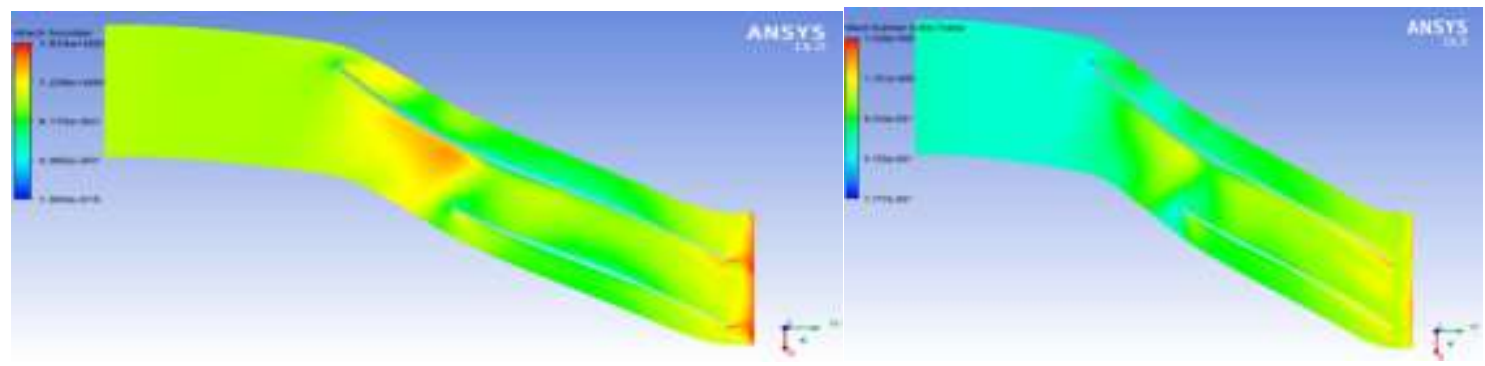

Figure 8: Mack number contour plot

\subsection{Analysis}

The primary goal of this research is to assess the current capabilities of CFD analysis tools to predict flows within centrifugal compressors across a wide range of off-design operating conditions. Following the on design analysis of the impeller, the problem of the simulation of the entire compressor stage under various operating conditions was tackled. 


\subsection{Noise Analysis}

This analysis has been supplied to assist in the evaluation of tonal noise levels generated by low speed fans (Mach Number less than 0.4). The equations were obtained from available literature, however some equations may have alternate definitions. It is your responsibility to verify the accuracy of these definitions.

\subsection{Noise input data}

This data is based on the input to the Fan Noise macro. To change the input values, select Turbo tab > Fan Noise macro, change parameters and select Calculate to re-generate the report.
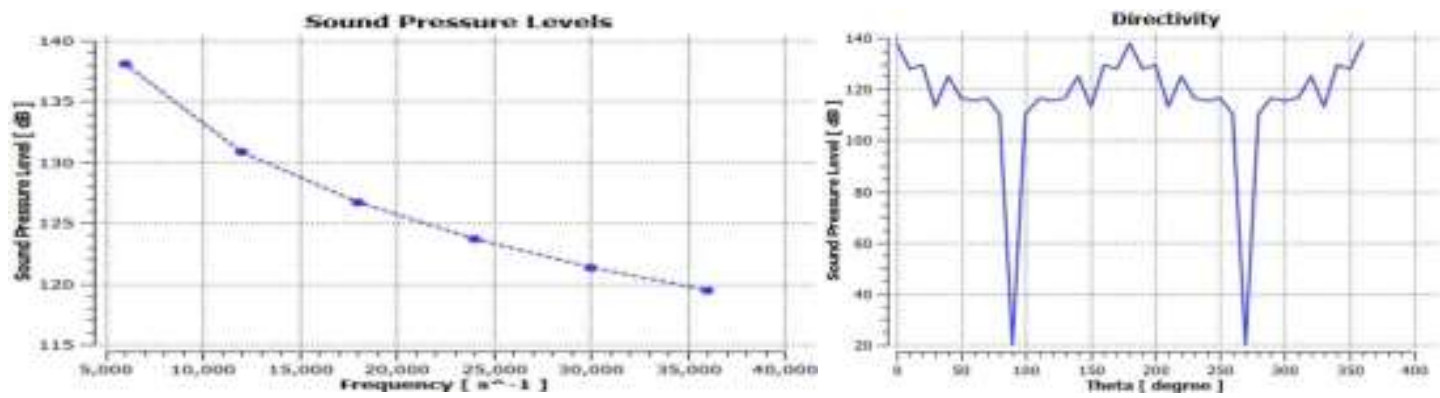

Figure 9: Sound Pressure and Directivity Levels

\subsection{Broadband Noise}

Broadband noise model is derived from Proudman's formula (see expression Proudman Sound Power Exp), which predicts overall sound power. Associated variable (Proudman Sound Power) is evaluated on the entire domain, allowing visualization of isosurfaces that can be used to locate the portion of the flow that is responsible for noise generation.Note that this model predicts overall noise levels, not at a specific observer location.

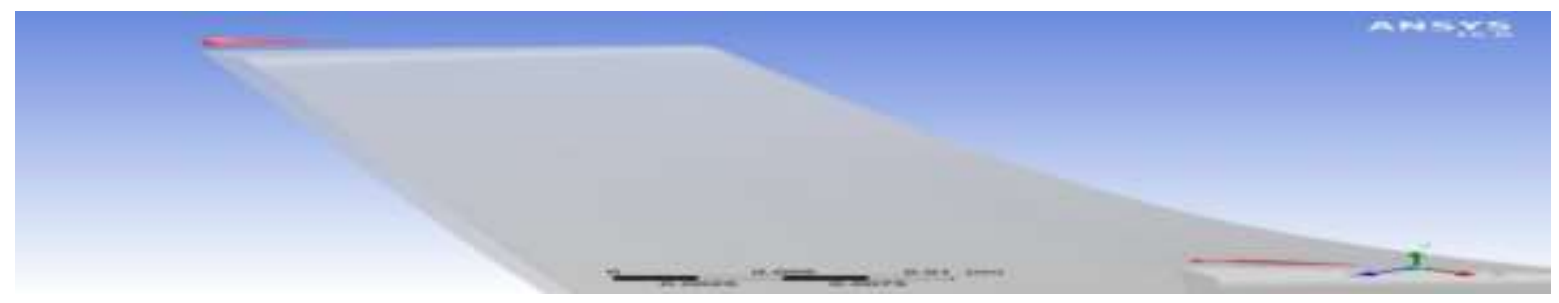

\subsection{Noise Sources}

Figure 10: Isosurface at 95\% of Proudman Sound Power

This section reports on Monopole, Dipole and Quadrupole noise sources, derived from Ffowcs Williams and Hawkings (FW-H) equations. These sources can be compared with each other and with the broadband noise to determine the dominant noise source in the design.Monopole source is related to the movement of the source surface. It defines the volume displacement of the source. It is usually called self noise. .Dipole source describes the interaction between the fluid and the surface of the source. It defines the loading fluctuations exerted on the surface. Quadrupole source is related to the turbulence fluctuation levels of the fluid. It is also called self noise.

Table 4: Summary of noise sources at the blade and at the final timestep

\begin{tabular}{|l|r|r|l|}
\hline & Monopole Source Strength & Dipole Source Strength & \\
\hline Minimum & 0.0 & -32493.5 & {$[\mathrm{~Pa}]$} \\
\hline Maximum & 1554.0 & 295921.0 & {$[\mathrm{~Pa}]$} \\
\hline Average & 1012.8 & 109204.0 & {$[\mathrm{~Pa}]$} \\
\hline
\end{tabular}




\section{RESULTS}

The CFD simulation provides a virtual image of the internal flow in the centrifugal compressor, allowing for the performance analysis of more complex phenomena. It was made to the flow from the input to the exit of a centrifugal compressor stage with all the components in place using CFD tools.To improve understanding of fluid flow through the centrifugal compressor stage, vector plots, contour plots, and stream line graphs are created. The impeller blade are rotating with 40000rpm subjected to the mass flow rate $3 \mathrm{~kg} / \mathrm{s}$ with the working pressure and Temperature at stagnation conditions $101.353 \mathrm{pa}$ and $288.15 \mathrm{k}$ respectively. The pressure loading over stream wise direction for the span of $70 \%$. The results of this CFD analysis reveal many opportunities for flow simulation and performance of the centrifugal compressor. Certain changes in velocity and pressure, in particular, identify places where energy is lost. The flow velocity pattern of this analysis shows a few key areas where energy is lost. Flow velocity clearly diminishes past the cut water, and flow re circulation occurs around the eye of the impeller. This recirculated fluid flow does not contribute to the compressor performance, and the adjustments should be made to recover this lost energy, such as changes to the shroud geometry in ANSYS Vista CCD.
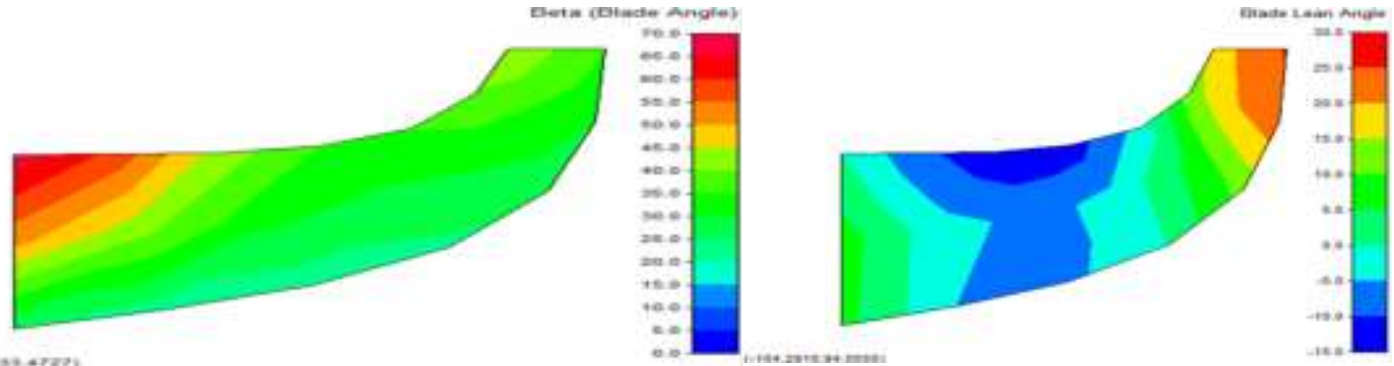

Figure 11: Beta location angle Contour Plot\&Blade lean Angle Contour Plot

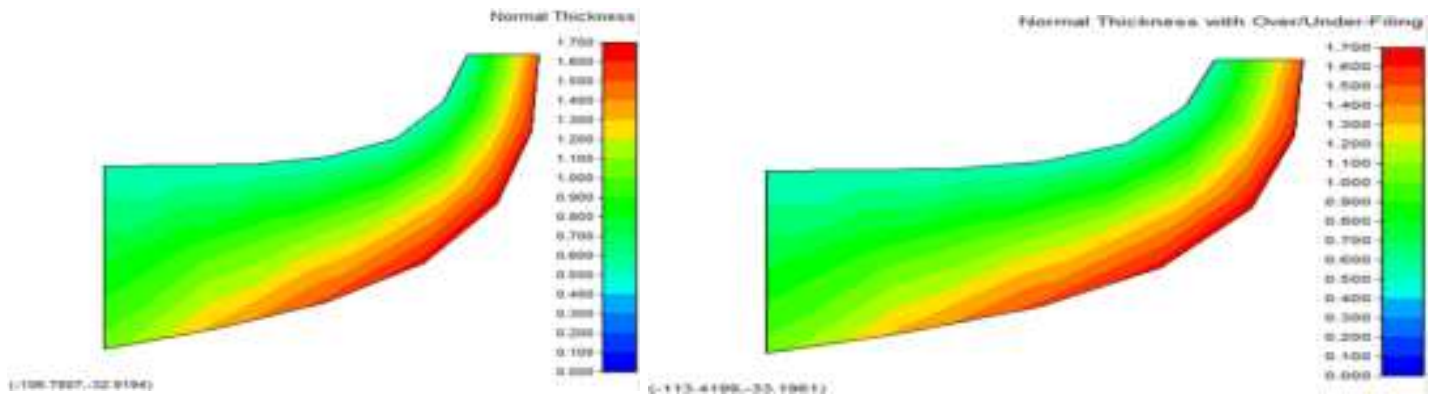

Figure 12: Normal thickness with over/under-filing

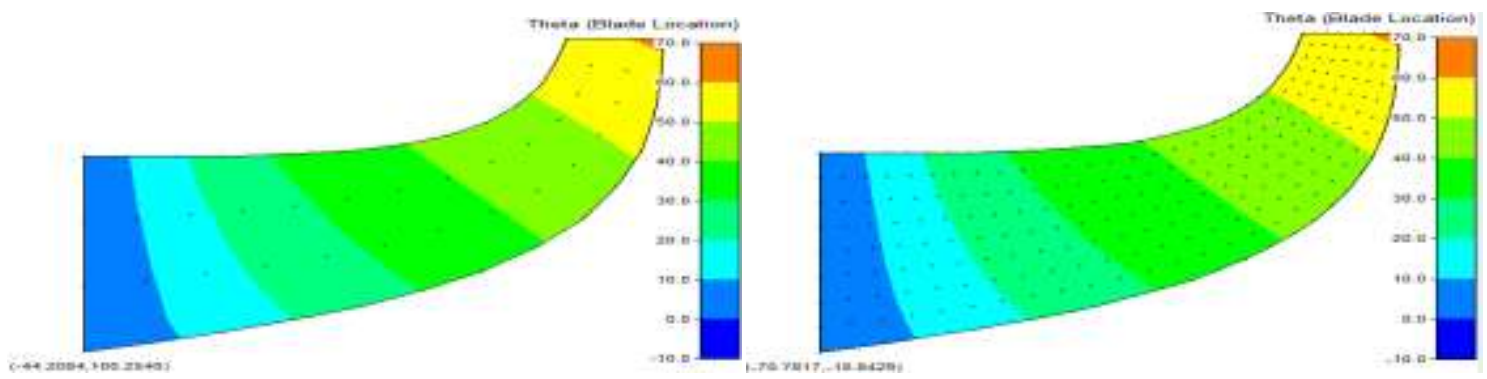

Figure 13: Grid density Course and fine contour plot 


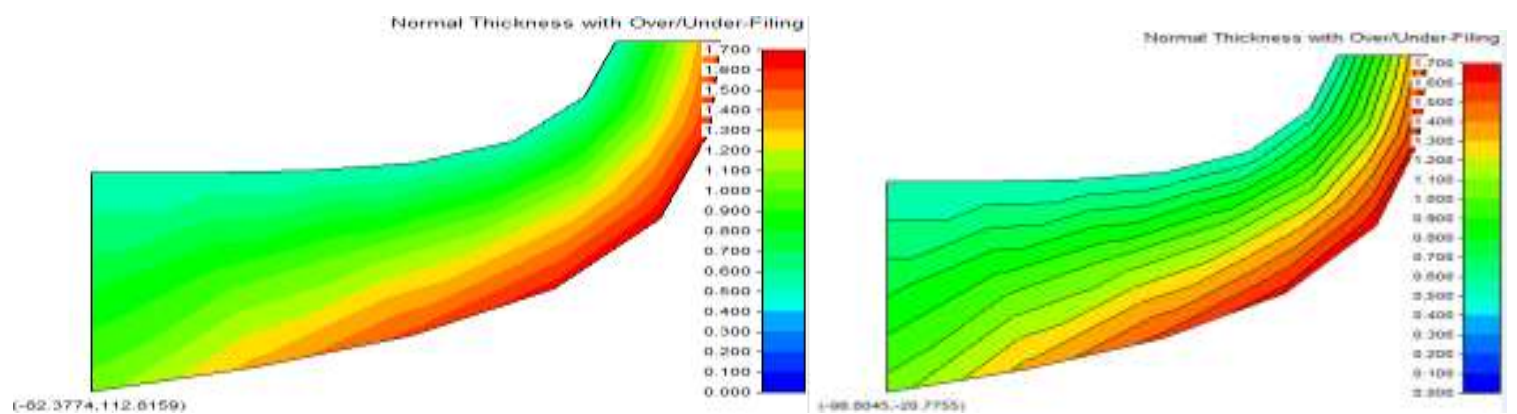

Figure 14 : Normal thickness of the grid density contour lines

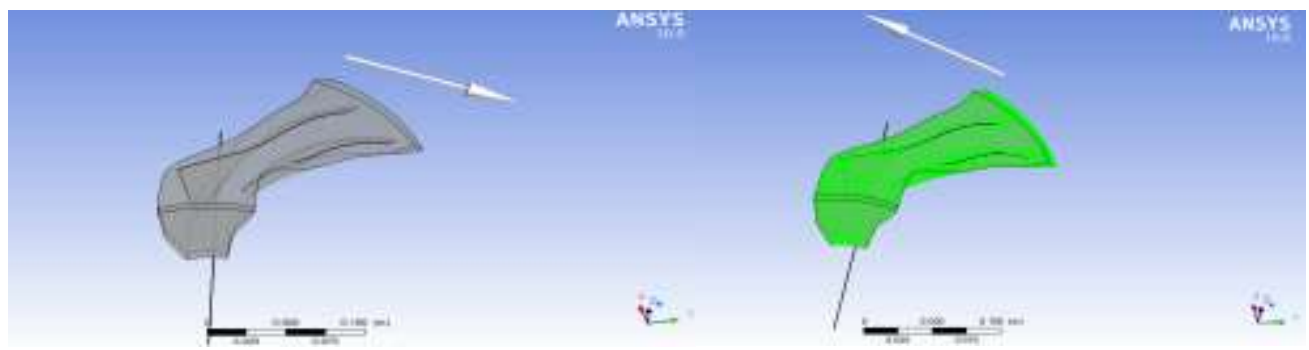

Figure 15. Direction of rotation with positive and negative

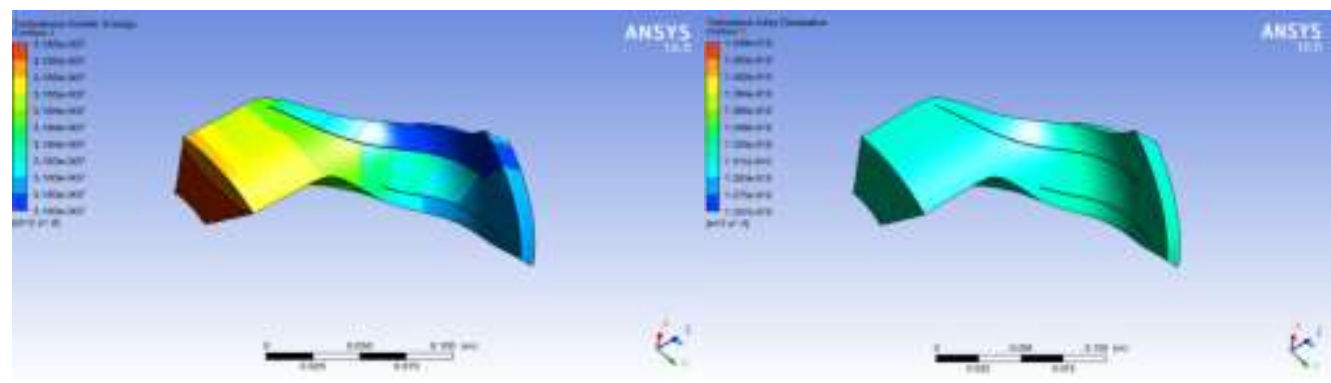

Figure 16: Turbulent Kinetic energy Vrs eddy dissipation contour plot
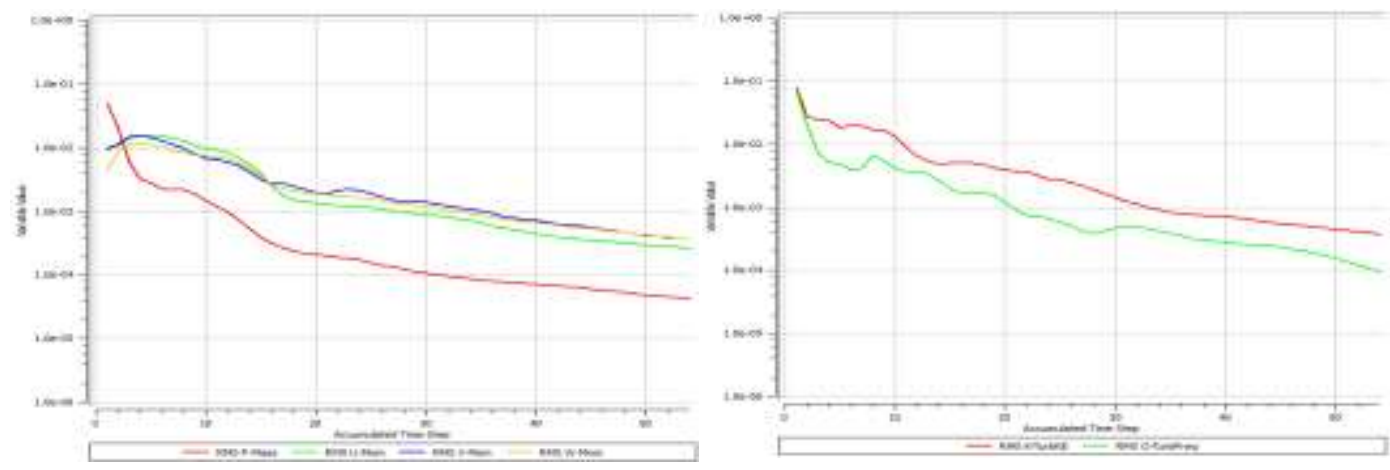

Figure 17 :Momentum, Heat transfer and Mass converged residual plot 


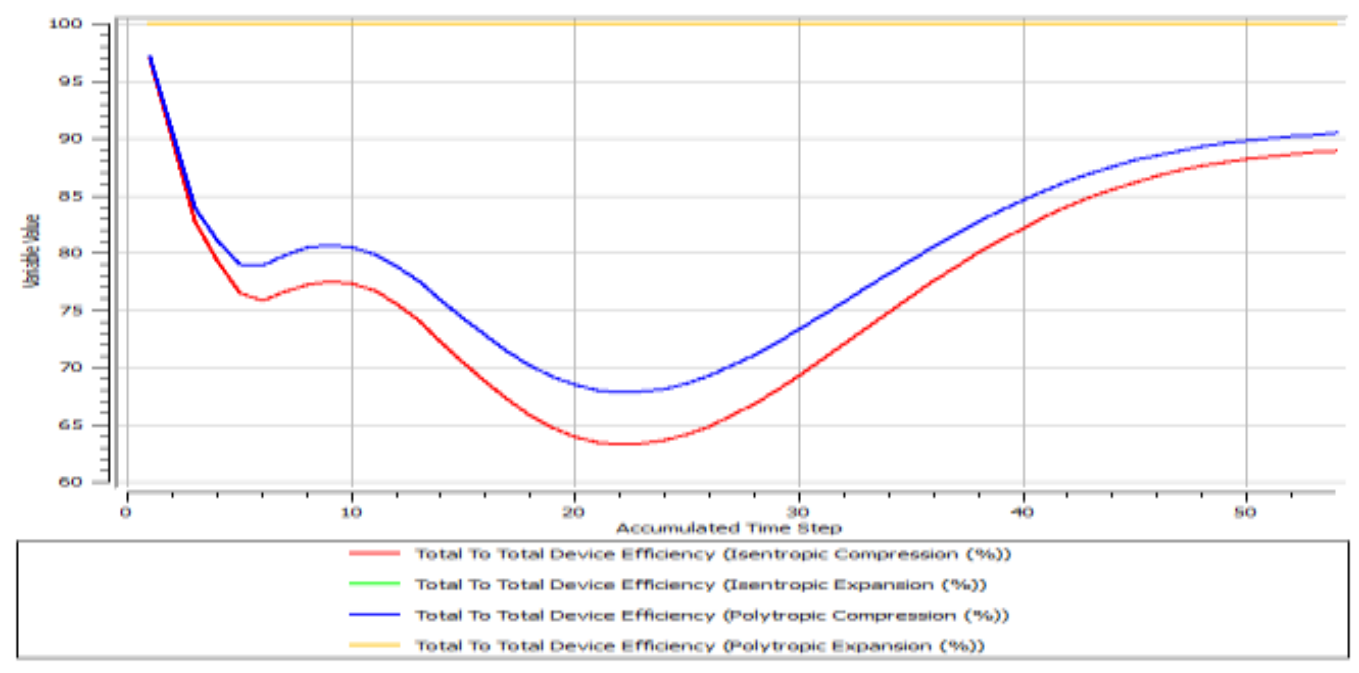

Figure 18: Efficiency curve of centrifugal compressor Compressor

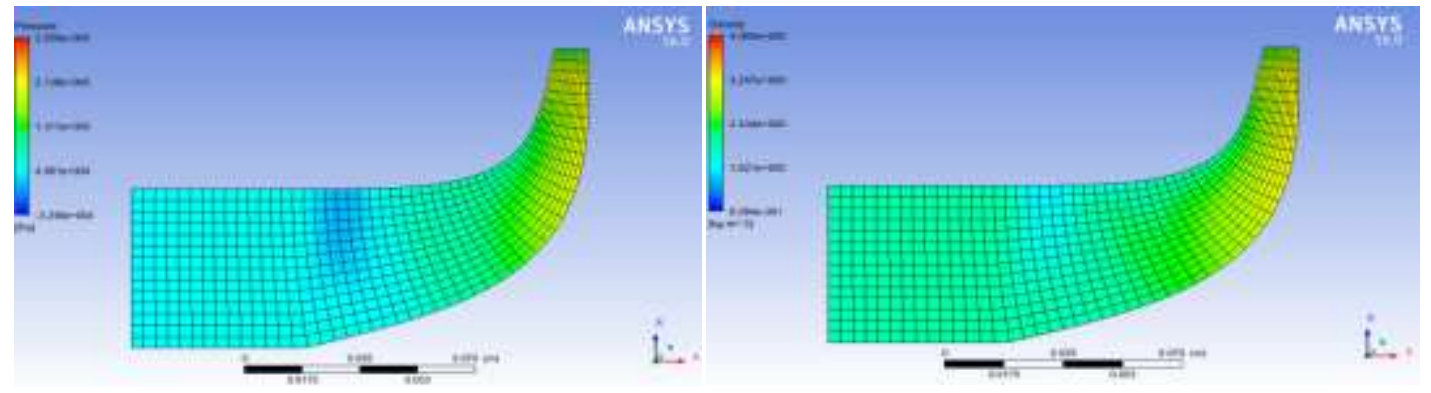

Figure 19: Meridional pressure and density

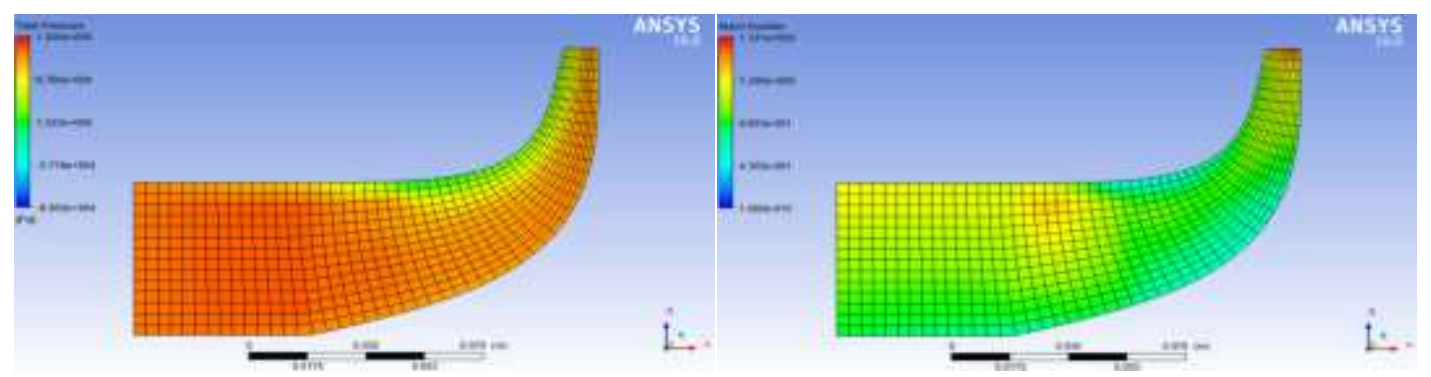

Figure 14: Meridional total pressure Vrs mach number 


\section{Reference}

[1] Kulkarni, V.V., Anil, T.R, Rajan, N.K..S., "An Impeller Blade Analysis of Centrifugal Gas Compressor Using CFD” International Journal in Engineering and Technology, 7(4):(2016).

[2] Boyce, M.P., Principles of Operation and Performance Estimation of Centrifugal Compressor,(1993).

[3] Wikipedia, the free encyclopedia, http://en.wikipedia.org/wiki/wind_power

[4] Metin Ozen, Ashok Das, Kim Parnell, “CFD Fundamental and Applications”, PP 7-8.

[5]Abdelmadjid, C., Mohamed, S. \& Boussad, B. (2013). CFD Analysis of the volute geometry effect on the turbulent air flow through the turbocharger compressor. Energy Procedia, 36, 746- 755.

[6] Azem, A., Mathis, P., Stute, F., Hoffmann, M., Muller, D. \& Hetzel, G. (2018). G. Efficiency increase of free running centrifugal fans through a pressure regain unit used in an air handling unit. Energy \& Buildings,165, 321-327.

[7] Danish, S. N., Khan, S. D.-D., Umer, U, Qureshi, S. R. \& Ma, C. (2014). Perfomance evaluation of tandem bladed centrifugal compressor. Engineering Applications of Computacional Fluid Mechanics, 8 (3), 382-295.

[8] Derakhshan, S., Yang, S.-S. \& Kong, F.-Y. (2012). Theoritical, numerical and experimental prediction of pump as turbine performance. Renewable Energy, 48, 507-513.

[9] Derakhsan, S., Pourmahdavi, M., Abdolahnejad, E., Reihani, A. \& Ojachi, A. (2013). Numerical shape optimization of a centrifugal pump impeller using artificial bee colony algorithm. Computers \& Fluids, 81. 145151. https://doi.org/10.1016/j.compfluid.2013.04.018.

[10] E. de M., Souza, J. S. de ., Magalhães, H. L. F., Porto, T. R. N. ., Silva, C. J. e ., Gomez, R. S., Lima, W. M. P. B. de, Lima, E. S. de, \& Lima, A. G. B. de. (2020). Oil-water separation process analysis in hydrocyclone via CFD. Research, Society and Development, 9 (11).

[11] Galloni, E., Parisi, P., Marignetti, F. \& Volpe, G. (20 a radial fan for electric motor cooling. Thermal Science and Engineering Progress, 8, 470-476. https://doi.org/10.1016/j.tsep.2018.10.003.

[12] Gjeta, A. (2019). Effect of Clearance Gap in Spiral Casing Design of a Centrifugal Fan with Optimized Impellers. European Journal of Engineering and Technology Research. 4 (9), 181-185.

[13] Hariharan, C. \& Govardhan, M. (2016). Improving performance of an industrial centrifugal blower with parallel wall volutes. Applied Thermal Engineering, 109 (part A), 53-64.

[14] Jafarzadech, B., Hajari, A., Alishahi, M. M. \& Akbari, M. H. (2011) The flow simulation of a low-specificspeed high-speed centrifugal pump. Applied Mathematical Modelling, 35, 241-249.

[15] J. H., Cha, K. H., Kim, K. Y., \& Jang, C. M. (2012). Numerical investigation on aerodynamic performance of a centrifugal fan with splitter blades. International Journal of Fluid Machinery and Systems, 5 (4), 168-173. 